Rapid Reviews COVID-19

\title{
Review 2: "Virtual reality \\ exercise to help COVID \\ patients with refractory \\ breathlessness"
}

Giuseppe Riva ${ }^{1}$

${ }^{1}$ Catholic University of Sacred Heart

Published on: Jan 25, 2022

License: Creative Commons Attribution 4.0 International License (CC-BY 4.0). 


\section{$\underline{\text { RR:C19 Evidence Scale rating by reviewer: }}$}

- Reliable. The main study claims are generally justified by its methods and data. The results and conclusions are likely to be similar to the hypothetical ideal study. There are some minor caveats or limitations, but they would/do not change the major claims of the study. The study provides sufficient strength of evidence on its own that its main claims should be considered actionable, with some room for future revision.

$* * * * * * * * * * * * * * * * * * * * * * * * * * * * * * * * * * * * * * *$

\section{Review:}

The use of immersive virtual reality (iVR) to improve breathing comfort in patients with refractory breathlessness $(\mathrm{RB})$ is timely, innovative, and has significant practical importance. In fact, given the diffusion of RB after severe COVID-19 pneumonia and its related disabilities that involve both the physical and the psychological/cognitive domain, easy-to-use, inexpensive and scientifically validated solutions aiming to reduce its effects are extremely necessary.

In general, I found the work - a controlled, randomized, single-blind, cross-over clinical study - well designed and carried out. The sample size, determined by power analysis, provides a reasonable estimate of the effects in question [1]. Moreover, the use of both synchronous (intervention) and asynchronous (control) feedback of the breathing in iVR guarantees that the effects are not due to a placebo effect.

Even if my general evaluation of the methodology is positive, there is still some space for improvement. First, the lack of any follow-up evaluation - clearly recognized by the authors as one of the study's main limitations - makes it impossible to verify if the effects of the training persist when patients are off-treatment. Second, the authors focused on qualitative data (primary outcomes) than on respiratory parameters (secondary outcomes). Finally, to be fully compliant with the open science standards, the availability of open data and open materials are needed, as well as a preregistered experiment and analysis plan.

Conceptually, the study is valuable because it extends iVR visual-respiratory stimulations from healthy subjects [2, 3] to clinical ones. Moreover, the study is significant because the observed effects are substantial: an improvement of $18 \%$ (about 1.3 points out of a 7-point Likert scale) in the level of breathing comfort and an 
improvement of $10 \%$ related to the experience of breathing agency. Finally, it uses a relatively inexpensive VR technology - a smartphone plus an enhanced cardboard headset - to achieve positive results.

Nevertheless, a key aspect of the study that suggests a space for future improvements is the lack of significant training effects on respiratory parameters. In another iVR study [3], using a more expensive VR setting, authors achieved a significant effect of the treatment on different respiratory parameters, including breathing wave amplitudes. A possible explanation for the lack of significant effects is the different view-point experienced by the user in the iVR training (allocentric - from outside - vs. the egocentric one - from inside -used in the previous research [3]). As demonstrated by different studies [4,5], using an allocentric viewpoint generates a significant reduction in one's cortical representations of the physical, embodied self, reducing our corporeal awareness and breathing control [3].

In conclusion, this is a reliable contribution to the literature suggesting that iVR can improve breathing comfort in patients recovering from severe COVID-19 pneumonia. As such, I recommend it for replication, extension, and further investigation. Options for future replication should include:

- a larger sample size

- a follow-up study to verify if the effects of the iVR training persist when patients are off-treatment;

- an improvement of the iVR training to achieve significant changes not only in the qualitative scores related to breathing comfort and agency but also in the objective respiratory parameters;

- a fully compliance with the open science standards through the availability of open data and open materials, as well as a preregistered experiment and analysis plan.

\section{References}

1. Hajian-Tilaki, K., Sample size estimation in diagnostic test studies of biomedical informatics. J Biomed Inform, 2014. 48: p. 193-204.

2. Adler, D., et al., Breathing and the Sense of Self: Visuo-Respiratory Conflicts Alter Body Self-Consciousness. Respiration, 2014. 87(6): p. 68-74. 
3. Monti, A., et al., The "embreathment" illusion highlights the role of breathing in corporeal awareness. Journal of Neurophysiology, 2020. 123(1): p. 420-427.

4. Eich, E., et al., Field and observer perspectives in autobiographical memory, in Social Thinking and Interpersonal Behavior, J.P. Forgas, K. Fiedler, and C. Sedikides, Editors. 2012, Taylor \& Francis: New York. p. 163-181.

5. Eich, E., et al., Neural systems mediating field and observer memories. Neuropsychologia, 2009. 47(11): p. 2239-51. 